\title{
Particle acceleration in a nearby galaxy cluster pair: the role of cluster dynamics ${ }^{\star}$
}

\author{
A. Botteon ${ }^{1,2,3}$, R. Cassano ${ }^{2}$, D. Eckert ${ }^{4}$, G. Brunetti ${ }^{2}$, D. Dallacasa ${ }^{1,2}$, T. W. Shimwell ${ }^{3,5}$, R. J. van Weeren ${ }^{3}$,
} F. Gastaldello ${ }^{6}$, A. Bonafede ${ }^{1,2,7}$, M. Brüggen ${ }^{7}$, L. Bîrzan ${ }^{7}$, S. Clavico ${ }^{8}$, V. Cuciti ${ }^{7}$, F. de Gasperin ${ }^{7}$, S. De Grandi ${ }^{8}$, S. Ettori ${ }^{9}, 10$, S. Ghizzardi ${ }^{6}$, M. Rossetti ${ }^{6}$, H. J. A. Röttgering ${ }^{3}$, and M. Sereno ${ }^{9}$

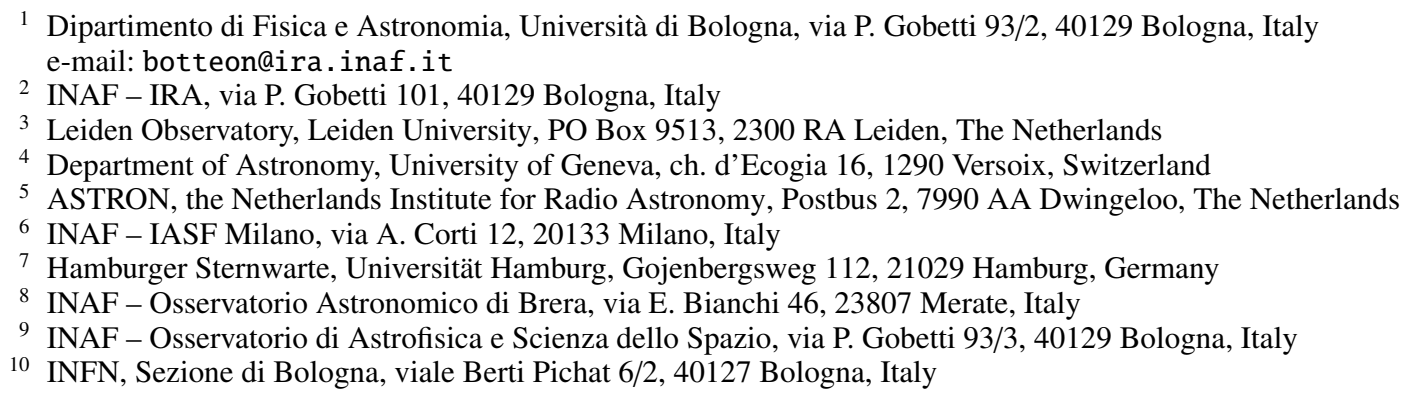

Received 5 June 2019 / Accepted 19 August 2019

\section{ABSTRACT}

\begin{abstract}
Context. Diffuse radio emission associated with the intracluster medium (ICM) is observed in a number of merging galaxy clusters. It is currently believed that a fraction of the kinetic energy in mergers is channeled into nonthermal components, such as turbulence, cosmic rays, and magnetic fields, which may lead to the formation of giant synchrotron sources in the ICM.

Aims. Studying merging galaxy clusters in different evolutionary phases is fundamental for understanding the origin of radio emission in the ICM.

Methods. We observed the nearby galaxy cluster pair RXC J1825.3+3026 $(z \sim 0.065)$ and CIZA J1824.1+3029 $(z \sim 0.071)$ at $120-168 \mathrm{MHz}$ with the LOw Frequency ARray (LOFAR) and made use of a deep (240 ks) XMM-Newton dataset to study the nonthermal and thermal properties of the system. RXC J1825.3+3026 is in a complex dynamical state, with a primary ongoing merger in the E-W direction and a secondary later stage merger with a group of galaxies in the SW, while CIZA J1824.1+3029 is dynamically relaxed. These two clusters are in a pre-merger phase.

Results. We report the discovery of a Mpc-scale radio halo with a low surface brightness extension in RXC J1825.3+3026 that follows the X-ray emission from the cluster center to the remnant of a galaxy group in the SW. This is among the least massive systems and the faintest giant radio halo known to date. In contrast to this, no diffuse radio emission is observed in CIZA J1824.1+3029, nor in the region between the pre-merger cluster pair. The power spectra of the X-ray surface brightness fluctuations of RXC J1825.3+3026 and CIZA J1824.1+3029 are in agreement with the findings for clusters exhibiting a radio halo and clusters where no radio emission has been detected, respectively.

Conclusions. We provide quantitative support to the idea that cluster mergers play a crucial role in the generation of nonthermal components in the ICM.
\end{abstract}

Key words. radiation mechanisms: non-thermal - radiation mechanisms: thermal - galaxies: clusters: intracluster medium galaxies: clusters: individual: RXC J1825.3+3026 - galaxies: clusters: individual: CIZA J1824.1+3029 - galaxies: clusters: general

\section{Introduction}

Galaxy clusters form through the continuous accretion of matter over cosmic time. During accretion, turbulent flows and shock waves are produced in the intracluster medium (ICM) and in some circumstances, they may generate cluster-scale synchrotron emission that is commonly referred to as giant radio halos or relics (e.g., van Weeren et al. 2019, for a recent review). The former category consists of apparently unpolarized sources

^ Reduced XMM and LOFAR images are only available at the CDS via anonymous ftp to cdsarc.u-strasbg. fr (130.79.128.5) or via http://cdsarc.u-strasbg.fr/viz-bin/cat/J/A+A/630/A77 found at the cluster center with a morphology similar to that of the X-ray emitting gas. The latter are elongated and often highly polarized at decimetric wavelengths and are located in the cluster outskirts. Both halos and relics are generally observed in massive dynamically disturbed clusters (e.g., Cassano et al. 2010a, 2013; Cuciti et al. 2015) and trace relativistic electrons and magnetic fields distributed in the ICM on Mpc-scales that are eventually (re)accelerated and amplified during merger events (e.g., Brunetti \& Jones 2014, for a review).

Observations at low radio frequencies with the LOw Frequency ARray (LOFAR) are revealing unprecedented details of the complex diffuse emission from the ICM (e.g., Bîrzan et al. 2019; Botteon et al. 2019; Clarke et al. 2019; Hoang et al. 2019a,b; 
Mandal et al. 2019; Savini et al. 2019; Wilber et al. 2019, for the most recent works). In particular, LOFAR is entering into unexplored territories for the study of nonthermal phenomena from the ICM, allowing us to explore the processes that are ongoing in the very early phases of a merger, well before the core crossing (Bonafede et al. 2018; Botteon et al. 2018, 2019; Govoni et al. 2019). Theoretical works show that equatorial shocks and turbulent flows should be excited in the ICM between pre-merging clusters; however, the kinetic energy associated with shock and turbulence at this phase is expected to be lower than in merging systems (e.g., Vazza et al. 2017; Ha et al. 2018), and it is unclear if a significant fraction of this energy can be channeled into nonthermal components already at this stage.

The galaxy clusters RXC J1825.3+3026 and CIZA J1824.1+3029 (hereafter RXCJ1825 and CIZAJ1824, respectively) constitute a binary system (mass ratio 1:1.6) at low redshift that is also known as Lyra complex (Clavico et al. 2019; Girardi et al. 2019). Due to its low Galactic latitude, this system has been challenging to observe, but the first focused X-ray (Clavico et al. 2019) and optical (Girardi et al. 2019) studies have recently been completed (see Table 1 for the main properties of the two clusters). However, to date, no targeted radio observations have been published. The picture emerging from both X-ray and optical data is that RXCJ1825 and CIZAJ1824 are gravitationally connected but do not have interacted yet, that is, they are in a pre-merger phase. Whereas CIZAJ1824 is dynamically relaxed, RXCJ1825 (i.e., the most massive cluster of the system) shows clear signatures of ongoing merging: primarily, two brightest cluster galaxies at the center and an irregular X-ray morphology (Clavico et al. 2019). In addition to this main merger, a minor collision also occurred in the SW periphery of RXCJ1825, where an extension of the X-ray emission is found between RXCJ1825 and the Southern Galaxy (SG in Fig. 1, right panel). This is an elliptical galaxy showing evidence for a $\sim 1 \mathrm{keV}$ "corona" surrounded by slightly hotter gas $(\sim 2 \mathrm{keV})$, suggesting that this is the remnant of a group of galaxies that was ram pressure stripped by the interaction with RXCJ1825 and caused the X-ray surface brightness extension in the SW (Clavico et al. 2019). All these features point toward a complex system involving multiple components, making this target an excellent candidate to search for diffuse synchrotron sources in the ICM at different dynamical stages. In particular, we are observing i) a relaxed cluster (CIZAJ1824), ii) an ongoing merger (RXCJ1825, in the E-W), iii) a pre-merger (the pair RXCJ1825/CIZAJ1824), and iv) a post-merger (RXCJ1825 and a group of galaxies, in the $\mathrm{SW}$ ).

In this paper, we report on the results from deep radio observations targeting the Lyra complex using the LOFAR High Band Antennas (HBA). We assume a $\Lambda$ CDM cosmology with $\Omega_{\Lambda}=$ $0.7, \Omega_{\mathrm{m}}=0.3, H_{0}=70 \mathrm{~km} \mathrm{~s}^{-1} \mathrm{Mpc}^{-1}$, and adopt the convention $S_{v} \propto v^{-\alpha}$ for radio synchrotron spectrum afterward.

\section{Observations and data reduction}

\subsection{LOFAR}

The cluster pair RXCJ1825/CIZAJ1824 was observed with LOFAR in HBA_DUAL_INNER mode on 21 June 2018 (project code: LC10_013). The observation was performed following the scheme of the LOFAR Two-meter Sky Survey (LoTSS; Shimwell et al. 2017), that is, $8 \mathrm{~h}$ on-source time bookended by two 10 min scans of the flux calibrator 3C295 using LOFAR HBA operating in the $120-168 \mathrm{MHz}$ frequency band. The $48 \mathrm{MHz}$ bandwidth is centered at the central frequency
Table 1. Properties of RXCJ1825 and CIZAJ1824.

\begin{tabular}{lrr}
\hline \hline & RXCJ1825 & CIZAJ1824 \\
\hline Redshift & 0.065 & 0.071 \\
Right ascension $(\mathrm{h}, \mathrm{m}, \mathrm{s})$ & 182520.0 & 182406.8 \\
Declination $\left({ }^{\circ},{ }^{\prime},{ }^{\prime \prime}\right)$ & +302611.2 & +302932.5 \\
$M_{500}\left(10^{14} \mathrm{M}_{\odot}\right)$ & $4.08 \pm 0.13$ & $2.46 \pm 0.63$ \\
$r_{500}(\mathrm{kpc})$ & $1105 \pm 12$ & $932 \pm 79$ \\
$L_{500}\left(10^{44} \mathrm{erg} \mathrm{s}^{-1}\right)$ & $2.42 \pm 0.02$ & $0.74 \pm 0.02$ \\
$\langle k T\rangle(\mathrm{keV})$ & $4.86 \pm 0.05$ & $2.14 \pm 0.05$ \\
Scale $\left(\mathrm{kpc} \operatorname{arcsec}^{-1}\right)$ & 1.248 & 1.354 \\
\hline
\end{tabular}

Notes. Redshifts are taken from Girardi et al. (2019), while $M_{500}$ and $r_{500}$ are derived from the deprojected mass profiles and are taken from Ettori et al. (2019, RXCJ1825) and Clavico et al. (2019, CIZAJ1824). The luminosity within $r_{500}$ in the $0.1-2.4 \mathrm{keV}$ band $L_{500}$ and the average temperature $\langle k T\rangle$ is from Clavico et al. (2019).

$144 \mathrm{MHz}$, which is also the reference frequency for the LOFAR images shown here.

We make use of the direction-dependent data reduction pipeline $^{1}$ v2.2 developed by the LOFAR Surveys Key Science Project. This latest version of the pipeline includes improvements in the calibration and imaging of extended sources and is currently adopted to process LoTSS observations (see Sect. 5 in Shimwell et al. 2019), and it will be thoroughly discussed in Tasse et al. (in prep.). The data processing exploits PREFACTOR (van Weeren et al. 2016; Williams et al. 2016; de Gasperin et al. 2019), KILlMS (Tasse 2014a,b; Smirnov \& Tasse 2015), and DDFACET (Tasse et al. 2018) to perform direction-independent and direction-dependent calibration and imaging of the entire LOFAR field of view (FoV). To improve the image quality toward the target field, we subtracted from the $u v$ data all the sources in the LOFAR FoV using the models derived from the pipeline, except for those in a $38.3^{\prime} \times 38.3^{\prime}$ region that contains RXCJ1825 and CIZAJ1824. Then, we performed additional phase and amplitude self-calibration loops to correct the residual artifacts in the small extracted region where the directiondependent errors are assumed to be negligible. The details of this extraction and recalibration step will be discussed in a forthcoming paper (van Weeren et al., in prep.).

Images at different resolutions were produced with WSCLEAN v2.6 (Offringa et al. 2014) using the Briggs weighting scheme (Briggs 1995) with robust $=-0.5$ and suitable tapering of the visibilities. An inner $u v$ cut of $80 \lambda$, corresponding to an angular scale of $43^{\prime}$, was applied to the data to drop the shortest spacings where calibration is more challenging. The LOFAR high- and medium-resolution images of the clusters are shown in Fig. 1.

Because of inaccuracies in the LOFAR HBA beam model, the LOFAR flux density scale can show systematic offsets (see van Weeren et al. 2016; Hardcastle et al. 2016). We checked and corrected the flux density scale by comparing the brightest compact sources extracted from the TIFR GMRT Sky Survey (TGSS; Intema et al. 2017) with the LOFAR image. Throughout the paper, we applied the correction factor computed from the mean LOFAR/TGSS integrated flux density ratio of 1.15 ; uncertainties on the LOFAR flux densities are dominated by the calibration error of $20 \%$, which was adopted in agreement with LoTSS measurements (Shimwell et al. 2019).

1 https://github.com/mhardcastle/ddf-pipeline 

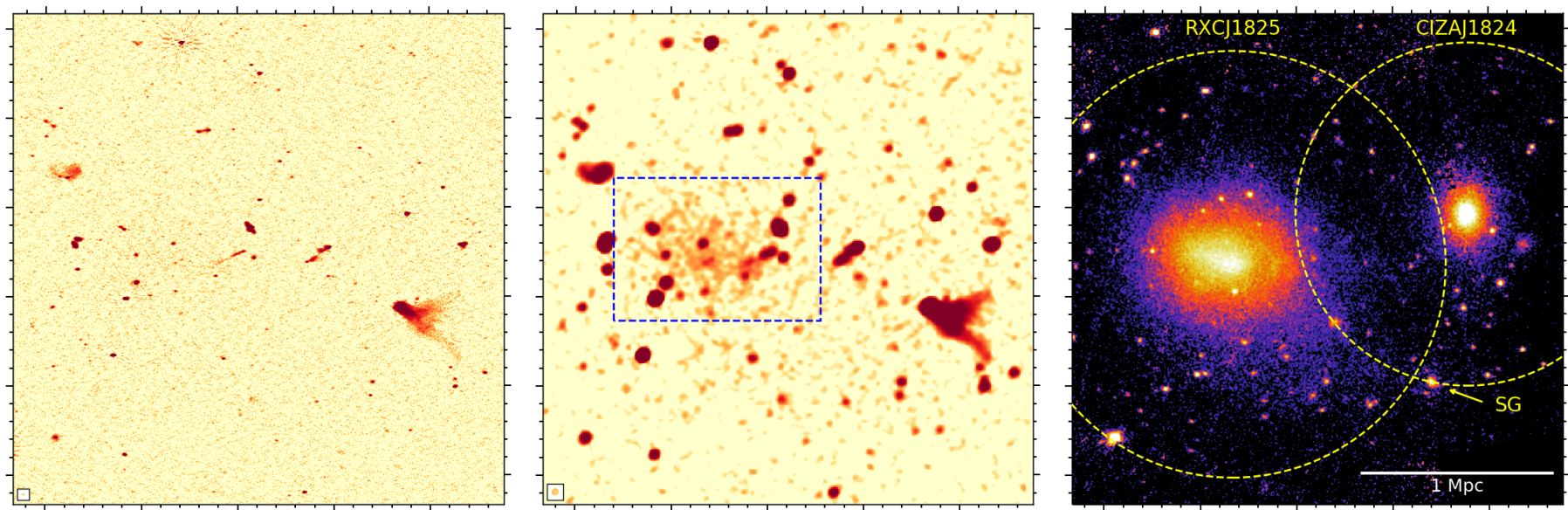

Fig. 1. Cluster pair RXCJ1825/CIZAJ1824 as observed with LOFAR HBA at high (left) and medium (center) resolution, and with XMM-Newton in the $0.5-2.0 \mathrm{keV}$ band (right). The resolution and rms noise of the LOFAR images are $8.5^{\prime \prime} \times 4.7^{\prime \prime}$ and $\sigma=110 \mu \mathrm{Jy} \mathrm{beam}{ }^{-1}(\mathrm{high})$, and $27.1^{\prime \prime} \times 24.4^{\prime \prime}$ and $\sigma=220 \mu \mathrm{Jy}_{\text {beam }}^{-1}$ (medium). The beam sizes are shown in the bottom left corners. The blue box in the LOFAR medium-resolution image shows the region where we evaluate the flux density of the halo. Yellow circles in the XMM-Newton image denote the approximate location of $r_{500}$ for each cluster (cf. Table 1), while the arrow indicates the Southern Galaxy. The displayed images have matched coordinates and cover a FoV of $33^{\prime} \times 33^{\prime}$ (cf. Fig. 2)

\subsection{XMM-Newton}

RXCJ1825 was observed in the context of the XMM-Newton Cluster Outskirts Project (X-COP; Eckert et al. 2019; Ettori et al. 2019; Ghirardini et al. 2019) for a total exposure time of $240 \mathrm{ks}$ divided into two central pointings and four offset pointings, one of which contains CIZAJ1824. We retrieved and processed these observations following standard data reduction recipes of the XMM-Newton Extended Source Analysis Software (ESAS; Snowden et al. 2008). In Fig. 1 (right panel) we show the XMMNewton background-subtracted and exposure-corrected mosaic image in the $0.5-2.0 \mathrm{keV}$ band of the cluster pair. For more details about the X-ray data analysis and interpretation, we refer to Clavico et al. (2019).

\section{Results}

\subsection{Diffuse emission in the ICM}

The LOFAR high-resolution image of the Lyra complex shows numerous radio galaxies with a wide range of morphologies (Fig. 1, left panel). The most prominent is the tailed radio galaxy that is located between CIZAJ1824 and the Southern Galaxy. This is discussed in Sect. 3.2. At a resolution of $27.1^{\prime \prime} \times 24.4^{\prime \prime}$ (Fig. 1, central panel), diffuse radio emission from the ICM of RXCJ1825 is clearly observed. The source follows the X-ray thermal emission and is slightly elongated in the E-W direction. Because of its location in the system, morphology, and cluster-size extent, we classify this source as a giant radio halo. In contrast, most of the radio emission from the vicinity of CIZAJ1824 is associated with the brightest cluster galaxy at its center, as commonly observed in cool-core systems. At this medium resolution, no significant radio emission is detected between RXCJ1825, CIZAJ1824, and the Southern Galaxy.

To better recover the emission from the radio halo in RXCJ1825 and study its connection with the thermal gas, we produced a LOFAR low-resolution image by applying a Gaussian $u v$ taper of $40^{\prime \prime}$ and then convolving it with a Gaussian beam of $60^{\prime \prime} \times 60^{\prime \prime}(\sim 75 \mathrm{kpc} \times 75 \mathrm{kpc})$ in the image plane. In Fig. 2 (left panel) we show the LOFAR low-resolution contours overlaid on the XMM-Newton image. This image shows the radio halo emission at the cluster center more clearly and

also suggests a low surface brightness extension from the halo toward the Southern Galaxy. The halo has a projected size of $1.0 \times 0.8 \mathrm{Mpc}$ when we take the radio emission above the $3 \sigma$ level into account. The halo extension toward the Southern Galaxy is patchy at this confidence level; the $1.5 \sigma$ contours highlight the underlying low surface brightness extension of emission better, which follows the X-ray morphology of the cluster remarkably well (Fig. 2, left panel). To improve the signal-to-noise ratio of this feature, we produced a lower resolution image using a Gaussian beam of $90^{\prime \prime} \times 90^{\prime \prime}(\sim 112 \mathrm{kpc} \times 112 \mathrm{kpc})$. Fig. 2 (right panel) shows the LOFAR very low-resolution contours where the extension of the radio halo toward the Southern Galaxy in the SW is detected at $3 \sigma$. The linear extent of the diffuse radio emission in this direction is up to $\sim 1.8 \mathrm{Mpc}$. In Sect. 4 we discuss the possible origin of this extension; it is likely related to merger between RXCJ1825 and a galaxy group. Finally, we note that in the LOFAR low-resolution images of Fig. 2, the radio emission located between RXCJ1825 and CIZAJ1824 is related to a double radio galaxy (cf. Fig. 1, left panel).

We evaluate the flux density of the radio halo in the blue box in Fig. 1 (central panel), which roughly covers the $3 \sigma$ contour of Fig. 2 (left panel). We adopted the two following procedures. As a first approach, we subtracted the clean component models of the discrete sources observed at high resolution in Fig. 1 (left panel) from the visibilities, re-imaged the new dataset, and evaluated the flux density in the source-subtracted images. In this case, the halo flux density is $S_{144}=153 \pm 31 \mathrm{mJy}$ at $144 \mathrm{MHz}$ and is consistent at medium and low resolution.

As a second approach, we measured the flux density in the blue box of Fig. 1 (central panel) taking both discrete sources and diffuse emission into account, and then subtracted the flux densities of the 18 discrete sources measured in the high-resolution image. This step is useful to evaluate the accuracy of the subtraction of radio galaxies with extended structures ( 3 out 18 , accounting for $\sim 208 \mathrm{mJy}$ ), such as those observed in the direction of RXCJ1825. The total (discrete sources+diffuse) flux density is $\sim 885 \mathrm{mJy}$, consistently derived in the mediumand low-resolution images. By subtracting the flux density of $\sim 712 \mathrm{mJy}$ due to the 18 embedded sources, a flux density of $S_{144}=173 \pm 35 \mathrm{mJy}$ is associated with the ICM emission. This value is higher than that found in the first approach and 

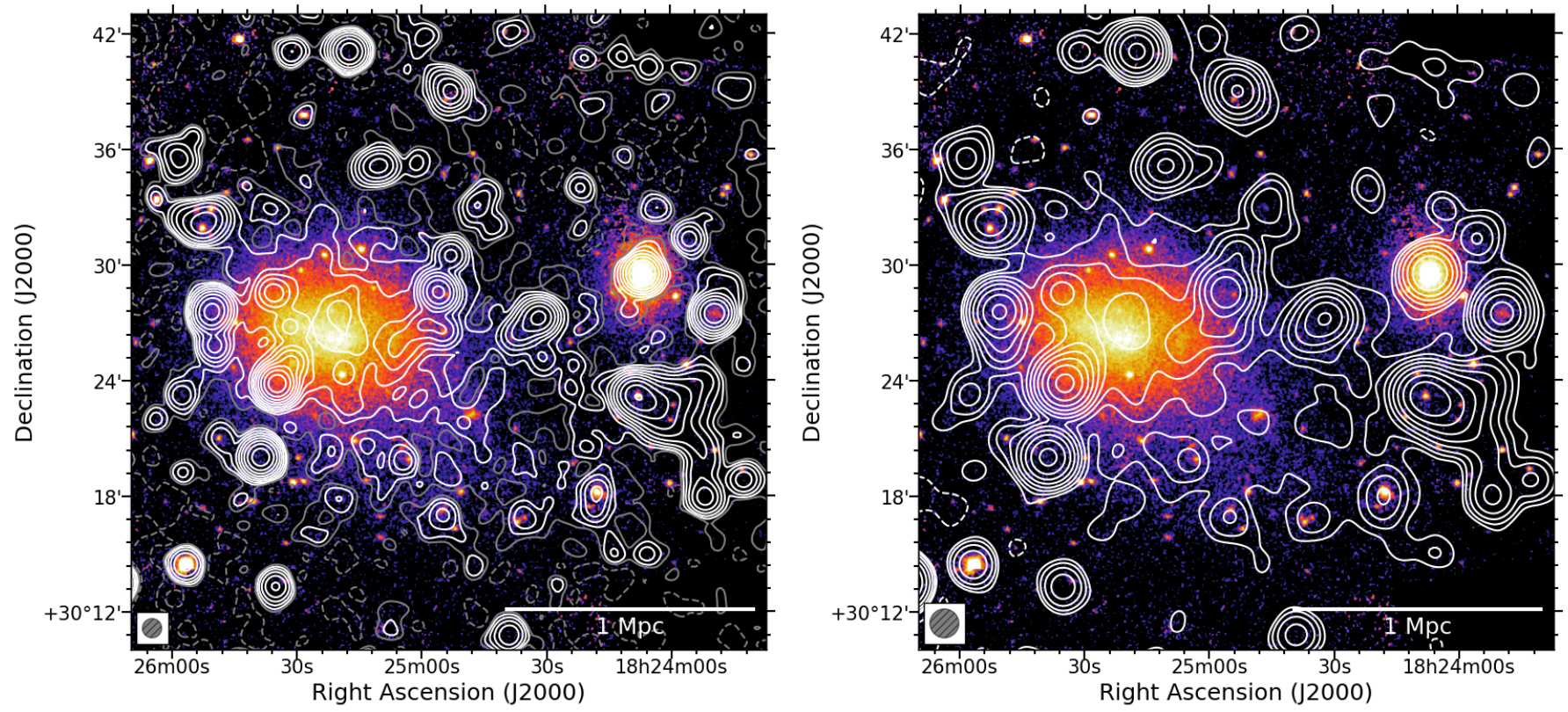

Fig. 2. LOFAR radio contours overlaid on the XMM-Newton color image. Left: low-resolution $\left(60^{\prime \prime} \times 60^{\prime \prime}\right)$ contours spaced by a factor of 2 starting from $1.5 \sigma$ (the first contour is reported in gray), where $\sigma=300 \mu \mathrm{Jy} \mathrm{beam}^{-1}$. The negative $-1.5 \sigma$ contours are shown as dashed gray lines. Right: very low-resolution $\left(90^{\prime \prime} \times 90^{\prime \prime}\right)$ contours spaced by a factor of 2 starting from $3 \sigma$, where $\sigma=415 \mu \mathrm{Jy} \mathrm{beam}^{-1}$. The negative $-3 \sigma$ contours are shown as dashed lines. The beams are displayed in the bottom left corners.

highlights the complexity of subtracting the extended emission of the discrete sources embedded in the radio halo.

For the reminder of this paper, we adopt the average flux density value of $S_{144}=163 \pm 47 \mathrm{mJy}$ for the radio halo in RXCJ1825. The radio power at $144 \mathrm{MHz}$ is $P_{144}=(1.7 \pm 0.5) \times$ $10^{24} \mathrm{~W} \mathrm{~Hz}^{-1}$. When we assume a typical halo spectral index of $\alpha=1.3$ (e.g., Feretti et al. 2012, and references therein), the expected flux density at $1.4 \mathrm{GHz}$ is $S_{1.4}=8.5 \pm 2.5 \mathrm{mJy}$, implying a radio power of $P_{1.4}=(8.7 \pm 2.5) \times 10^{22} \mathrm{~W} \mathrm{~Hz}^{-1}$. This radio power is a factor of $2-4$ below the extrapolation at the cluster mass (or luminosity) of the best-fit $P_{1.4}-M_{500}$ (or $\left.P_{1.4}-L_{500}\right)$ relation of Cassano et al. (2013). The flux density values expected at $1.4 \mathrm{GHz}$ are below the sensitivity level of our reprocessed NRAO VLA Sky Survey (NVSS; Condon et al. 1998 ) images, which have an rms of $0.22 \mathrm{mJy}$ beam $^{-1}$. However, very short snapshots imply sparse $u v$ coverage which is known to prevent the detection of large-scale low surface brightness diffuse emission. Therefore we could not provide a meaningful constraint on the spectral index of the radio halo. Deep, pointed JVLA and/or uGMRT observations are planned to perform this type of analysis

To the best of our knowledge, RXCJ1825 is the least powerful giant radio halo discovered so far and one of the smallest clusters known to date to host this kind of diffuse emission. It probes regions in the $P_{1.4}-M_{500}$ and $P_{1.4}-L_{500}$ planes that are poorly constrained because of the limited sensitivity of previous instruments (e.g., Cassano et al. 2013; Bîrzan et al. 2019). LOFAR and future radio interferometers (such as the Square Kilometer Array) have the sensitivity to explore the lower end of the correlations and to test the theoretical models of radio halo formation in these regimes (e.g., Cassano et al. 2010b, 2012).

We can estimate an upper limit to the flux density of the nondetected diffuse emission in CIZAJ1824 via $S=A \times \sigma$ (e.g., Hoang et al. 2018). When we consider a conservatively large radio halo area of $A=250^{2} \pi \mathrm{kpc}^{2}$ and the noise of our low-resolution LOFAR image, we obtain an upper limit of $S_{144}<8.1 \mathrm{mJy}$ for the level of diffuse emission at the center of CIZAJ1824. Assuming, again, a spectral index index of $\alpha=1.3$, this would imply a radio power more than a factor of 10 below the extrapolation of the $P_{1.4}-M_{500}$ relation, underlying the dichotomy between RXCJ1825 (merger, with radio halo) and CIZAJ1824 (relaxed, without diffuse radio emission).

\subsection{Tailed radio galaxy}

A bright tailed radio galaxy is observed between CIZAJ1824 and the Southern Galaxy. Girardi et al. (2019) found that the host galaxy, which is cospatially coincident with the nucleus of the radio galaxy, has redshift $z=0.0699 \pm 0.0003$, hence it is part of the Lyra complex, whose mean redshift is $\langle z\rangle=0.0674 \pm 0.0003$ (based on 198 spectroscopically confirmed members of the system). The flux density of the source is $S_{144}=577 \pm 115 \mathrm{mJy}$.

Tailed radio galaxies are commonly divided into narrowangle and wide-angle sources, depending on the angle between the radio jets or lobes as they deploy in the ICM (e.g., Miley 1980). In the case shown in Fig. 3 , the nucleus of the radio galaxy is embedded in a cocoon of emission with a forked diffuse elongation. Because of the lack of high-resolution data, it is currently premature to classify this source into one of the two classes described above. Its morphology might be due to projection effects related to the motion of the host galaxy, which moves at large angle with respect to the plane of the sky (Girardi et al. 2019), similarly to the case of NGC 7385 (Rawes et al. 2015). A cartoon indicating the proposed scenario is shown in Fig. 3. Distorted radio galaxies can be generated by the interaction between the tails and the surrounding ICM, providing another demonstration of the complexity of the system.

\section{Discussion}

The detection of a radio halo in the dynamically disturbed cluster RXCJ1825 and the absence of diffuse radio emission in the coolcore system CIZAJ1824 strongly support the idea that merger 


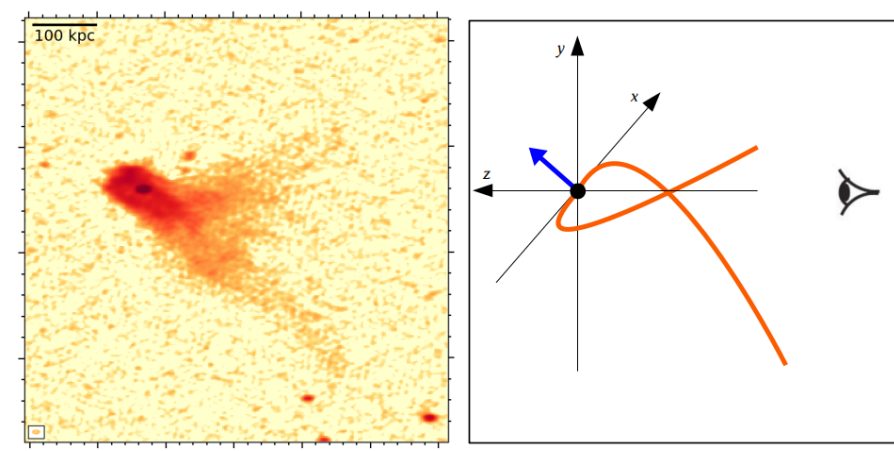

Fig. 3. Zoom-in of the tailed radio galaxy in Fig. 1 (left panel). A cartoon showing the projection that might explain the source morphology is also reported. The galaxy moves in the direction indicated by the blue arrow.

events play a fundamental role in the generation of nonthermal components in the ICM (e.g., Cassano et al. 2010a). The Fourier power spectrum analysis of the X-ray surface brightness fluctuations provides information on the Mach numbers of turbulent motions in the ICM (e.g., Gaspari \& Churazov 2013; Gaspari et al. 2014; Zhuravleva et al. 2014). We used the deep XMMNewton observation to extract the power spectra of RXCJ1825 and CIZAJ1824 within a radius of $200 \mathrm{kpc}$ from the X-ray peak following the method of Eckert et al. (2017). As shown in Fig. 4, the amplitude of gas density fluctuations $\delta \rho / \rho$ at the maximum scale of the main cluster RXCJ1825 is a factor of $\sim 2$ larger than that of the cool-core CIZAJ1824. Because the sound speed $c_{\mathrm{s}}$ in RXCJ1825 is a factor $\sim 1.5$ higher, this would imply that the 3D turbulent velocity dispersion $\sigma_{\mathrm{v}} \approx 3.7 c_{\mathrm{s}} \delta \rho / \rho$ of the main system is $\sim 3$ times higher than that of CIZAJ1824 (under the hypothesis of isotropic turbulent motions, see Gaspari \& Churazov 2013). The values of the 2D amplitude of RXCJ1825 and CIZAJ1824 at the largest scale are in line with those observed for radio halo and non-radio halo clusters, respectively, in the sample analyzed by Eckert et al. (2017).

The indication of the low surface brightness radio extension following the X-ray extension in the SW direction suggests past interaction between RXCJ1825 and the galaxy group that hosted the Southern Galaxy. This region and that between RXCJ1825 and CIZAJ1824 have been investigated in detail by Clavico et al. (2019) using the deep XMM-Newton observation ${ }^{2}$. Whereas no residual emission is observed in the region between RXCJ1825 and CIZAJ1824, the region between RXCJ1825 and the Southern Galaxy appears dynamically active. We extracted one-dimensional brightness profiles along the slices reported in Fig. 5 (left panels) using radio (discrete source subtracted) and X-ray images with a comparable resolution of $60^{\prime \prime}$ (e.g., Shimwell et al. 2016) to quantitatively show that there is a connection between the thermal and nonthermal emission of the ICM. The locations of the two slices were chosen to avoid contaminating sources in the X-ray image. Although the radio image is naturally more sensitive to local brightness variations (e.g., because of residual emission left over by subtracted discrete sources), the profiles of Fig. 5 (right panels) show an overall good match between the $\mathrm{X}$-ray and radio brightness distribution

\footnotetext{
2 In particular, they (i) modeled the surface brightness distribution of the two clusters assuming two elliptical single- $\beta$ model profiles and produced a residual image by comparing the difference pixel by pixel between data and model and (ii) created unsharp masked images with different Gaussian smoothing to search for surface brightness features in the ICM.
}

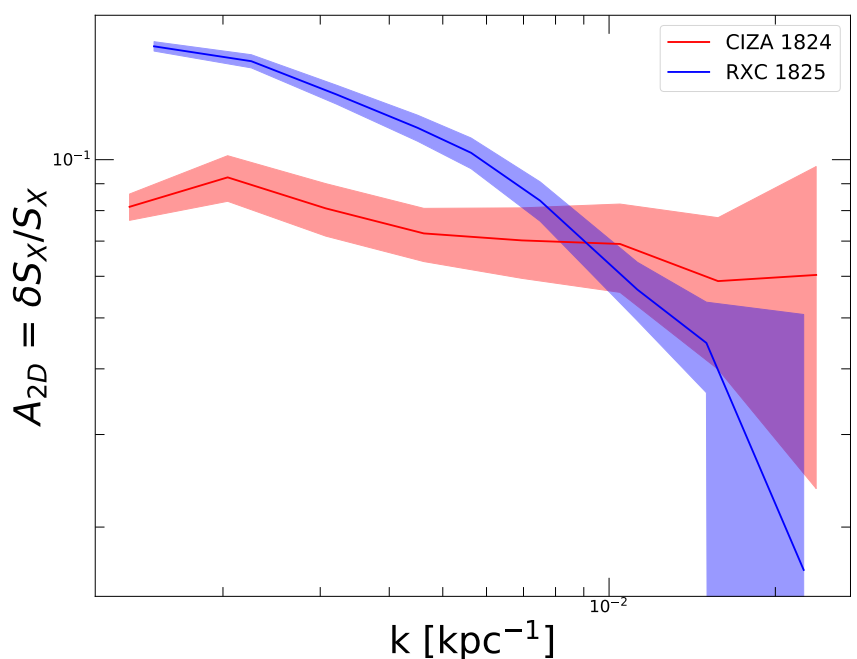

Fig. 4. Fractional amplitude of projected (2D) X-ray surface brightness fluctuations $A_{2 \mathrm{D}}=\left(P_{2 \mathrm{D}} 2 \pi k^{2}\right)^{1 / 2}$ for RXCJ1825 (blue) and CIZAJ1824 (red) as a function of wave number $k$. The power spectra $P_{2 \mathrm{D}}$ were extracted within a circle of $200 \mathrm{kpc}$ radius around the X-ray peak of both structures. The shaded areas show the $1 \sigma$ confidence intervals for both regions.

(except for the absence of radio emission from CIZAJ1824), indicating a tight connection between the two components and possibly a common origin. The X-ray extension toward the SW might be due to gas from the group of galaxies that hosted the Southern Galaxy in the past and that has been stripped by ram pressure during the interaction with RXCJ1825 or to gas belonging to RXCJ1825 generated by tidal interaction with the group. In the first case, the radio emission in this region may be explained as synchrotron emission by particles that are reaccelerated by the turbulence produced in the wake of the group during its motion toward the cluster outskirts. In the second case, the radio emission could result from the advection of the relativistic and thermal plasma from the cluster center to the SW as a result of the high-velocity motion of the galaxy group. The X-ray spectral analysis does not allow us to distinguish between these two possibilities because the temperature of the ICM surrounding the "corona" of the Southern Galaxy is consistent either with a temperature that once belonged to a group, or with that of RXCJ1825 at this radial distance (see Clavico et al. 2019).

Girardi et al. (2019) studied the merger kinematics between RXCJ1825 and CIZAJ1824 adopting the two-body model described in detail by Beers et al. (1982) and Gregory \& Thompson (1984) and concluded that the clusters are gravitationally bounded and very likely in an incoming orbit. The fact that no surface brightness nor temperature enhancement is observed in the X-rays between RXCJ1825 and CIZAJ1824 (Fig. 5) suggests that the gas between the clusters has not yet been compressed and heated, indicating that these objects are in a pre-merger phase. We do not find any evidence of diffuse radio emission between RXCJ1825 and CIZAJ1824 in our LOFAR data, confirming the picture drawn by optical and X-ray analyses. So far, possible diffuse radio emission in the ICM between two galaxy clusters has only been reported in the pairs A1758N-A1758S (Botteon et al. 2018) and A399-A401 (Govoni et al. 2019).

\section{Conclusions}

We have presented results from a LOFAR HBA observation of the low-redshift galaxy cluster pair RXCJ1825/CIZAJ1824. 

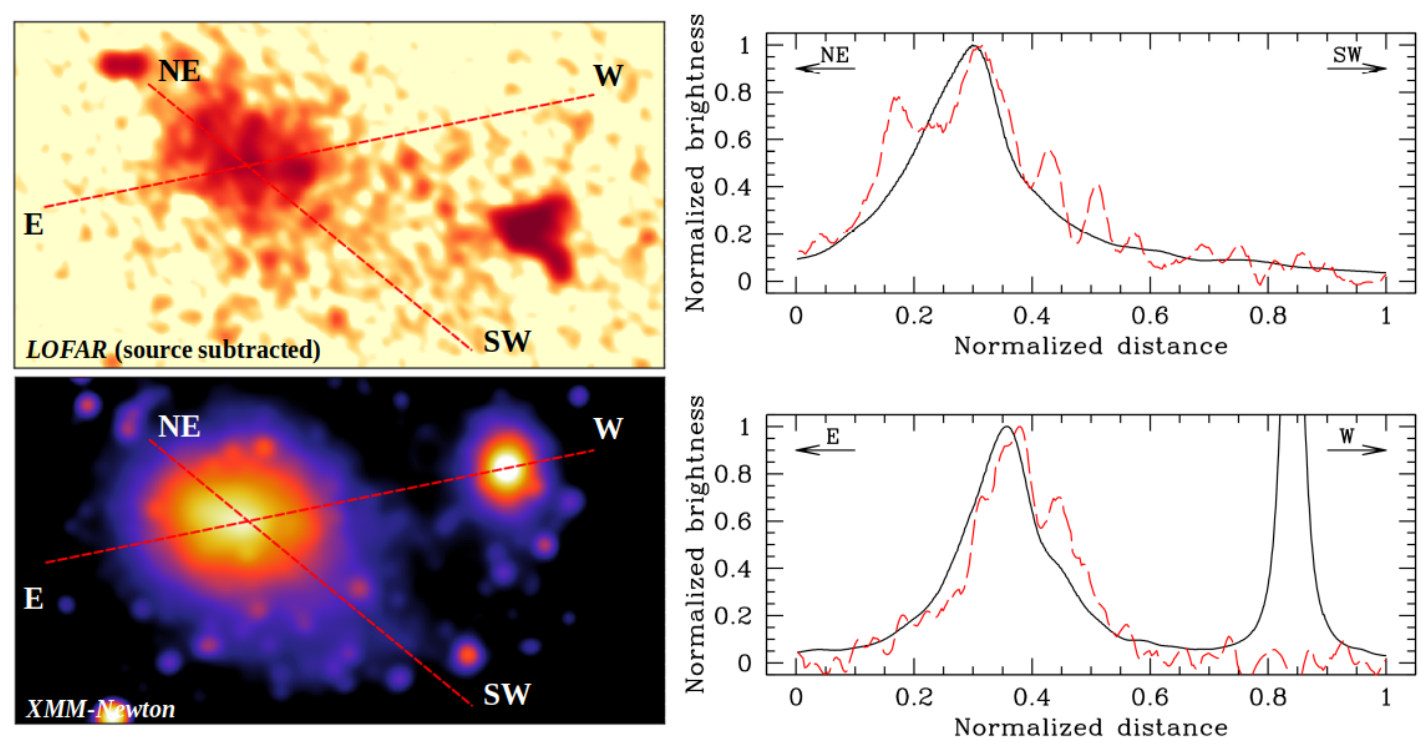

Fig. 5. One-dimensional brightness profiles of the X-ray (black lines) and radio emission (red dashed lines) extracted from the dashed lines reported in the left panels that display the LOFAR (discrete source subtracted) and XMM-Newton images convolved with a comparable resolution of 60". The profiles are normalized at the brightness peak of RXCJ1825 in each band.

Recent optical and X-rays studies showed that this system is in a pre-merger phase, CIZAJ1824 is a cool-core cluster, while RXCJ1825 is dynamically disturbed and is undergoing a main merger in the E-W direction and experienced an additional collision with a group of galaxies in the SW. Our findings are summarized below.

1. We discovered a giant radio halo in RXCJ1825. The halo has a projected size of $1.0 \mathrm{Mpc} \times 0.8 \mathrm{Mpc}$ and an integrated flux density in the range $S_{144}=163 \pm 47 \mathrm{mJy}$, corresponding to a radio power of $P_{144}=(1.7 \pm 0.5) \times 10^{24} \mathrm{~W} \mathrm{~Hz}^{-1}$.

2. RXCJ1825 is the least powerful radio halo know to date and one of the least massive systems hosting such an object. When a spectral index $\alpha=1.3$ is assumed, it would fall a factor of 2-4 below the extrapolation of the current $P_{1.4}-M_{500}$ relation in the low-mass regime.

3. The radio halo has a low surface brightness extension in the direction of the Southern Galaxy, leading to a maximum linear extent of the diffuse radio emission up to $\sim 1.8 \mathrm{Mpc}$. The remarkable spatial coincidence between the thermal and nonthermal emission indicates that this feature is a consequence of the energy dissipated on small scales due to the interaction between RXCJ1825 and a galaxy group.

4. The radio emission from CIZAJ1824 comes from the brightest cluster galaxy, as commonly observed in relaxed systems. The highly sensitive LOFAR observation has allowed us to place an upper limit for the diffuse radio emission a factor of 10 below the extrapolation of the $P_{1.4}-M_{500}$ relation.

5. No diffuse emission is detected between RXCJ1825 and CIZAJ1824, which are in a pre-merger phase.

Overall, the dichotomy observed between the dynamical states, radio properties, and power spectra of $\mathrm{X}$-ray surface brightness fluctuations of the two galaxy clusters paints a consistent picture connecting cluster mergers and the generation of diffuse radio emission in the ICM.

Our results show that LOFAR has the potential to detect new extended radio sources in the ICM in nearby and low-mass clusters that were previously missed by old-generation instruments and surveys. This allows us to open a new window into the study of diffuse emission in these systems that is crucial to con- strain the low-power and low-mass end of the $P_{1.4}-M_{500}$ scaling relation.

Acknowledgements. We thank M. Girardi and W. Boschin for sharing with us details of their optical analysis, and we acknowledge the anonymous referee for useful suggestions. RJvW acknowledges support from the VIDI research programme with project number 639.042.729, which is financed by the Netherlands Organisation for Scientific Research (NWO). ABon acknowledges financial support from the ERC-Stg DRANOEL, no 714245, and from the MIUR grant FARE SMS. SE acknowledges financial contribution from the contracts ASI 2015-046-R.0. SE and MS acknowledge financial contribution from the contract ASI-INAF n.2017-14-H.0. HJAR acknowledge support from the ERC Advanced Investigator programme NewClusters 321271. This paper is based (in part) on data obtained with the International LOFAR Telescope (ILT) under project code LC10_013. LOFAR (van Haarlem et al. 2013) is the LOw Frequency ARray designed and constructed by ASTRON. It has observing, data processing, and data storage facilities in several countries, which are owned by various parties (each with their own funding sources), and are collectively operated by the ILT foundation under a joint scientific policy. The ILT resources have benefitted from the following recent major funding sources: CNRS-INSU, Observatoire de Paris and Université d'Orléans, France; BMBF, MIWF-NRW, MPG, Germany; Science Foundation Ireland (SFI), Department of Business, Enterprise and Innovation (DBEI), Ireland; NWO, The Netherlands; The Science and Technology Facilities Council, UK; Ministry of Science and Higher Education, Poland; Istituto Nazionale di Astrofisica (INAF), Italy. This research made use of the Dutch national e-infrastructure with support of the SURF Cooperative (e-infra 180169) and the LOFAR e-infra group, and of the LOFAR IT computing infrastructure supported and operated by INAF, and by the Physics Dept. of Turin University (under the agreement with Consorzio Interuniversitario per la Fisica Spaziale) at the C3S Supercomputing Centre, Italy. The Jülich LOFAR Long Term Archive and the German LOFAR network are both coordinated and operated by the Jülich Supercomputing Centre (JSC), and computing resources on the Supercomputer JUWELS at JSC were provided by the Gauss Centre for Supercomputing e.V. (grant CHTB00) through the John von Neumann Institute for Computing (NIC). This work is also based on observations obtained with XMM-Newton, an ESA science mission with instruments and contributions directly funded by ESA Member States and NASA. This research made use of APLpy, an open-source plotting package for Python (Robitaille \& Bressert 2012).

\section{References}

Beers, T., Geller, M., \& Huchra, J. 1982, ApJ, 257, 23

Bîrzan, L., Rafferty, D., Cassano, R., et al. 2019, MNRAS, 487, 4775

Bonafede, A., Brüggen, M., Rafferty, D., et al. 2018, MNRAS, 478, 2927 Botteon, A., Shimwell, T., Bonafede, A., et al. 2018, MNRAS, 478, 885 
A. Botteon et al.: Particle acceleration in a nearby galaxy cluster pair: the role of cluster dynamics

Botteon, A., Shimwell, T., Bonafede, A., et al. 2019, A\&A, 622, A19

Briggs, D. 1995, in American Astronomical Society Meeting Abstracts, Bull. Am. Astron. Soc., 27, 1444

Brunetti, G., \& Jones, T. 2014, IJMPD, 23, 30007

Cassano, R., Ettori, S., Giacintucci, S., et al. 2010a, ApJ, 721, L82

Cassano, R., Brunetti, G., Röttgering, H., \& Brüggen, M. 2010b, A\&A, 509, A68

Cassano, R., Brunetti, G., Norris, R., et al. 2012, A\&A, 548, A100

Cassano, R., Ettori, S., Brunetti, G., et al. 2013, ApJ, 777, 141

Clarke, A., Scaife, A., Shimwell, T., et al. 2019, A\&A, 627, A176

Clavico, S., De Grandi, S., Ghizzardi, S., et al. 2019, A\&A, submitted [arXiv:1908.02276]

Condon, J., Cotton, W., Greisen, E., et al. 1998, AJ, 115, 1693

Cuciti, V., Cassano, R., Brunetti, G., et al. 2015, A\&A, 580, A97

de Gasperin, F., Dijkema, T., Drabent, A., et al. 2019, A\&A, 622, A5

Eckert, D., Gaspari, M., Vazza, F., et al. 2017, ApJ, 843, L29

Eckert, D., Ghirardini, V., Ettori, S., et al. 2019, A\&A, 621, A40

Ettori, S., Ghirardini, V., Eckert, D., et al. 2019, A\&A, 621, A39

Feretti, L., Giovannini, G., Govoni, F., \& Murgia, M. 2012, A\&ARv, 20, 54

Gaspari, M., \& Churazov, E. 2013, A\&A, 559, A78

Gaspari, M., Churazov, E., Nagai, D., Lau, E., \& Zhuravleva, I. 2014, A\&A, 569, A67

Ghirardini, V., Eckert, D., Ettori, S., et al. 2019, A\&A, 621, A41

Girardi, M., Boschin, W., De Grandi, S., et al. 2019, A\&A, submitted [arXiv:1908.02277]

Govoni, F., Orrù, E., Bonafede, A., et al. 2019, Science, 364, 981

Gregory, S., \& Thompson, L. 1984, ApJ, 286, 422

Ha, J.-H., Ryu, D., \& Kang, H. 2018, ApJ, 857, 26

Hardcastle, M., Gürkan, G., van Weeren, R., et al. 2016, MNRAS, 462, 1910
Hoang, D., Shimwell, T., van Weeren, R., et al. 2018, MNRAS, 478, 2218

Hoang, D., Shimwell, T., van Weeren, R., et al. 2019a, A\&A, 622, A20

Hoang, D., Shimwell, T., van Weeren, R., et al. 2019b, A\&A, 622, A21 Intema, H., Jagannathan, P., Mooley, K., \& Frail, D. 2017, A\&A, 598, A78

Mandal, S., Intema, H., Shimwell, T., et al. 2019, A\&A, 622, A22

Miley, G. 1980, ARA\&A, 18, 165

Offringa, A., McKinley, B., Hurley-Walker, N., et al. 2014, MNRAS, 444, 606

Rawes, J., Worrall, D., \& Birkinshaw, M. 2015, MNRAS, 452, 3064

Robitaille, T., \& Bressert, E. 2012, Astrophysics Source Code Library [record ascl:1208.017]

Savini, F., Bonafede, A., Brüggen, M., et al. 2019, A\&A, 622, A24

Shimwell, T., Luckin, J., Brüggen, M., et al. 2016, MNRAS, 459, 277

Shimwell, T., Röttgering, H., Best, P., et al. 2017, A\&A, 598, A104

Shimwell, T., Tasse, C., Hardcastle, M., et al. 2019, A\&A, 622, A1

Smirnov, O., \& Tasse, C. 2015, MNRAS, 449, 2668

Snowden, S., Mushotzky, R., Kuntz, K., \& Davis, D. 2008, A\&A, 478, 615

Tasse, C. 2014a, ArXiv e-prints [arXiv:1410.8706]

Tasse, C. 2014b, A\&A, 566, A127

Tasse, C., Hugo, B., Mirmont, M., et al. 2018, A\&A, 611, A87

van Haarlem, M., Wise, M., Gunst, A., et al. 2013, A\&A, 556, A2

van Weeren, R., Williams, W., Hardcastle, M., et al. 2016, ApJS, 223, 2

van Weeren, R., de Gasperin, F., Akamatsu, H., et al. 2019, Space Sci. Rev., 215, 16

Vazza, F., Jones, T., Brüggen, M., et al. 2017, MNRAS, 464, 210

Wilber, A., Brüggen, M., Bonafede, A., et al. 2019, A\&A, 622, A25

Williams, W., van Weeren, R., Röttgering, H., et al. 2016, MNRAS, 460, 2385

Zhuravleva, I., Churazov, E., Schekochihin, A., et al. 2014, ApJ, 788, L13 